\title{
COMPARISON OF ARTHROCENTESIS PLUS PLATELET RICH PLASMA WITH ARTHROCENTESIS ALONE IN THE TREATMENT OF TEMPOROMANDIBULAR JOINT DYSFUNCTION
}

\author{
Humaira Sarwar, Irfan Shah, Ali Akhtar Khan, Muhammad Afzal*, Adnan Babar, Amir Mushtaq Baig \\ Armed Forces Institute of Dentistry/National University of Medical Sciences (NUMS) Rawalpindi Pakistan, *21 MDC, Quetta Pakistan
}

\begin{abstract}
Objective: To evaluate the role of combination therapy of platelet rich plasma (PRP) with arthrocentesis and to compare it with arthrocentesis alone in the temporomandibular dysfuction (TMD) patients.

Study Design: Quasi experimental study.

Place and Duration of Study: Oral and Maxillofacial Surgery department, Armed Forces Institute of Dentistry, Combined Military Hospital Rawalpindi, from Jan 2017 to Jun 2019.

Methodology: Forty-two patients diagnosed with refractory unilateral temporomandibular dysfuction, were included in the study. Patients were divided in two groups with 21 temporomandibular joints in each. Arthrocentesis alone was the control group (group I) and arthrocentesis with intra-articular injection of platelet rich plasma (group II) was the study group. Treatment outcomes were assessed and compared for all patients based on clinical parameters of pre and post treatment; for pain, maximal mouth opening and temporomandibular joint clicking sounds.

Results: Out of 42 patients, 33 (79\%) were females and 9 (21\%) were males with mean age of $34.3 \pm 8.4$ years. There was statistically significant difference in both groups for all variables. The $p$-value of maximum mouth opening of both groups before and after treatment was 0.746 and 0.01 , joint clicking sounds were present in $69 \%$ of our patients before the treament and it reduced to $14 \%$ after the treatment. There was marked gradual decrease in pain of both groups, group I (6.48 \pm 1.470 to $1.81 \pm$ $0.602)$ and group II $(7.29 \pm 1.007$ to $1.19 \pm 0.402)$.

Conclusion: Combination therapy of platelet rich plasma with arthrocentesis is more effective treatment method than arthrocentesis alone for chronic temporomandibular dysfuctions.
\end{abstract}

Keywords: Arthrocentesis, Combination therapy, Platelet rich plasma, Temporomandibular joint dysfunction.

This is an Open Access article distributed under the terms of the Creative Commons Attribution License ( https://creativecommons.org/licenses/by-nc/4.0/), which permits unrestricted use, distribution, and reproduction in any medium, provided the original work is properly cited.

\section{INTRODUCTION}

Temporomandibular joint dysfunction (TMD) is an acute or chronic non-odontogenic pain of the orofacial region ${ }^{1}$. It is one of the most commonly presenting chronic orofacial pains, more frequently occurring in females ${ }^{2,3}$. It is a multifactorial condition characterized by debilitating muscular and joint pain, clicking sounds, limited mouth opening and functional limitations of the jaw ${ }^{1,3,4}$.

Conservative and surgical therapeutic options are available for TMDs. The primary treatment goal of TMDs is to improve mandibular function, to relieve pain and improve quality of life. Conservative treatment modalities are the first line treatment and surgical intervention is indicated only in patients not responding to them ${ }^{1}$. Surgical interventions range from minimally invasive procedures like arthrocentesis, arthroscopy and intra-articular injections of platelet rich plasma to complex open joint surgical procedures ${ }^{3,4,5}$.

Within regenerative medicine filed, platelet rich

Correspondence: Dr Adnan Babar, Department of Dentistry, Armed Forces Institute of Dentistry, Rawalpindi Pakistan

Received: 18 Sep 2020; revised received: 18 Oct 2020; accepted: 04 Nov 2020 plasma (PRP) is among many of the new developments. It is used to reduce post-operative pain in total knee arthroplasty, epicondylitis, ligament and connective tissue injuries and also in neuropathic pain conditions with promising results ${ }^{6,7}$. PRP was first introduced in oral surgery procedures by Whitman et al ${ }^{4}$. It is used as a healing aide on oral surgical procedures including bone grafts, implants and maxillofacial reconstructions, sinus lift procedures, ridge augmentation, alveolar cleft repair and oral/nasal fistula repair ${ }^{5}$.

As PRP has resulted in improved outcomes in other fields, its use in maxillofacial surgery for TMD patients was studied to find its impact in this field. The objective of the study was to evaluate and compare the outcomes of arthrocentesis with PRP and arthrocentesis alone in the treatment of TMD based on clinical parameters.

\section{METHODOLOGY}

This was a quasi-experimental study carried out from January 2017 to June 2019. Sample was estimated using $G^{*}$ power calculator that a total of 42 patients would be required for the detection of a difference between groups using a two-tailed a of 0.05 and a power 
of 0.80 , while the means for two groups as reported in Rajput et $a l^{3}$, was as follow: mean and SD for group I = $39.2 \pm 6.2$, mean and SD for group II $=42.5 \pm 8.1$.

It was a quasi experimental study, conducted at Oral and Maxillofacial Surgery department department, Armed Forces Institute of Dentistry, Rawalpindi, from January 2017 to June 2019 after approval of Ethics Review Committee (905/Trg-ABPIK2) and taking informed consent from all participants of the study. Total 42 patients were included in this study, simple random table sampling technique was used and they were categorized into two groups, group I was control group (arthrocentesis) and group II was study group (arthrocentesis with PRP).

Each group included of 21 patients, who were initially managed with conservative therapy for TMDs, but their symptoms persisted. All patients of both genders, within age range of 20-60 years with unilateral TMD complaints having good general health and who were physically and psychologically able to undergo arthrocentesis were included in the study.

Patients with bilateral TMDs or TMDs due to reduced facial height after the loss of posterior teeth, patients having associated systemic bone or joint diseases (osteoarthritis, rheumatoid arthritis, and osteoporosis) and those taking any bone supplements were excluded from this study. Patients with history of any other TMJ pathology or surgery were also not considered for the study. Patients who were not considered medically fit for the use of PRP (platelet function disorder, fibrinogen deficiency, anti-coagulant treatment) were also excluded. Patient's age, gender, time since TMDs, maximal mouth opening, clicking sounds were recorded. Pain on mouth opening and chewing was measured on visual analogue scale (VAS). All assessments were recorded by same maxillofacial surgeon on each follow up visit. Follow up was done after one week and monthly for consecutive six months.

Patients were prepared for arthrocentesis after auriculotemporal nerve block. A straight line was drawn from earlobe to lateral canthus of eye. According to Nitzan et al ${ }^{8}$ method, first point was $10 \mathrm{~mm}$ anterior and $2 \mathrm{~mm}$ below cantho tragus line. Second point was $10 \mathrm{~mm}$ anterior from first point and $10 \mathrm{~mm}$ below the reference line. Overlying skin was disinfected after marking and patient was asked to open mouth as wide as possible. One 20 gauge needle was inserted at both points. Out of $300 \mathrm{ml}$ normal saline was injected under pressure through first needle to superior joint space and second needle provided the outflow. Mandible was gently manipulated in all positions to free up disc. After arthrocentesis, all patients in both groups were given one $\mathrm{ml}$ of triamcinolone $40 \mathrm{mg}$ injection intraarticulary.

PRP for group II patients was prepared by collection of peripheral blood from ulnar vein of patient. It was transferred to test tubes with sodium citrate (3.2\%) as anti-coagulant and centrifuged at $3200 \mathrm{rpm}$ for 12 minutes after placing even number of tubes in centrifuge rotor. PRP was formed above the erythrocyte layer. It was aspirated into separate syringe with caution ${ }^{6,9}$, and one $\mathrm{ml}$ was injected into the superior joint space after the arthrocentesis. Post operative antibiotics (Amoxiclav 625mg) twice daily for three days and NSAIDS (naproxen sodium) twice daily for one week were prescribed. Patients were informed for transient swelling over the joint with unpleasant sense of fullness and compression.

Data was collected using a specifically designed proforma. All patients clicking sounds, pain score on VAS and MMO was recorded before and after the treatment. Chi-square test was used to analyze clicking sounds in both groups and independent t-test was used to compare the pain and MMO using SPSS-20. A $p$-value of $\leq 0.05$ was considered statistically significant.

\section{RESULTS}

In this study total 42 patients were included. Out of 42 patients, $33(79 \%)$ were females and $9(21.4 \%)$ were males. The mean age of all patients was $34.35 \pm$ 8.46 with range of 21-52 years.

Among our patients, 11 (26.2\%) had symptomatic TMJ for less than three months, 21 (50\%) patients had TMD's complaints for 3-6 months, $6(14.3 \%)$ of our patients had symptoms for 6-12 months and 4 (9.5\%) were chronic cases of more than a year. Our patients were divided into group I and II. Group I included 21 TMJs, the mean age was $35 \pm 10$ years. In group II, the mean age was $34 \pm 7$ years (figure).

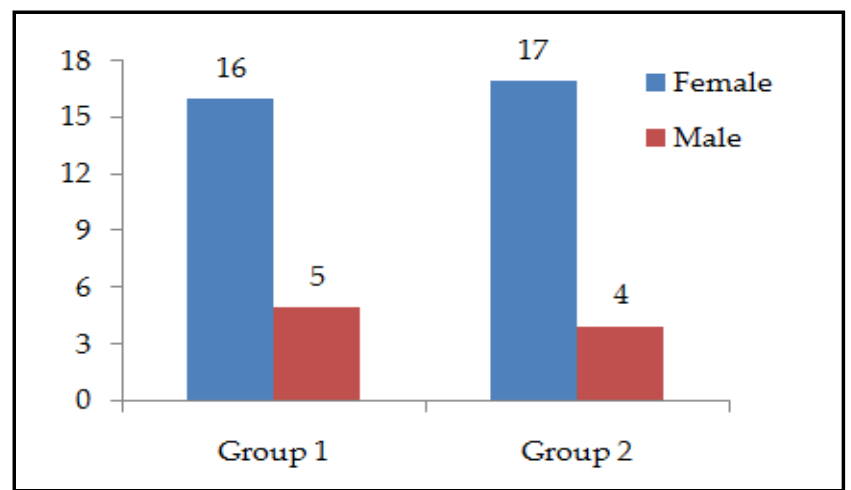

Figure: Gender distribution in both groups. 
Joint clicking was present in 29 (69\%) patients and $13(31 \%)$ had no clicking sound on mouth opening or closure before the treatment. After the arthrocentesis and arthrocentesis with PRP joint clicking sounds was found in $6(14 \%)$ of our patients and $36(86 \%)$ had no clicking sound on their last follow up. Effects of treatment on clicking sound are given in table-I. The mean values of both pre-operative and post-operative pain and $\mathrm{MMO}$ are in table-II.

Table-I: Outcomes of treatment on clicking sounds.

\begin{tabular}{|c|c|c|c|c|}
\hline \multirow{2}{*}{\multicolumn{2}{|c|}{ Groups }} & \multicolumn{3}{|c|}{ Pre-Operative } \\
\hline & & Present & \multicolumn{2}{|c|}{ Absent } \\
\hline \multicolumn{2}{|c|}{ Group I $(n=21)$} & $8(38 \%)$ & \multicolumn{2}{|c|}{$13(62 \%)$} \\
\hline \multicolumn{2}{|c|}{ Group II $(n=21)$} & $21(100 \%)$ & \multicolumn{2}{|c|}{ 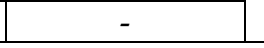 } \\
\hline \multicolumn{5}{|c|}{ Table- II: Outcomes of management. } \\
\hline \multicolumn{3}{|c|}{ Pre-Operative } & \multicolumn{2}{|c|}{ Post-Operative } \\
\hline Groups & & $p$-value & & $p$-value \\
\hline \multicolumn{5}{|l|}{ Pain } \\
\hline Group I & $\begin{array}{l}6.48 \pm \\
1.470 \\
\end{array}$ & \multirow[b]{2}{*}{0.07} & $\begin{array}{l}1.81 \pm \\
0.602 \\
\end{array}$ & \multirow{2}{*}{0.121} \\
\hline Group II & $\begin{array}{l}7.29 \pm \\
1.007\end{array}$ & & $\begin{array}{l}1.19 \pm \\
0.402\end{array}$ & \\
\hline \multicolumn{5}{|c|}{ Maximum Mouth Opening } \\
\hline Group I & $\begin{array}{c}12.81 \pm \\
2.857\end{array}$ & \multirow[b]{2}{*}{0.746} & $\begin{array}{c}31.24 \pm \\
5.7\end{array}$ & \multirow{2}{*}{0.01} \\
\hline Group II & $\begin{array}{c}12.71 \pm \\
3.068\end{array}$ & & $\begin{array}{c}39.43 \pm \\
3.28\end{array}$ & \\
\hline
\end{tabular}

\section{DISCUSSION}

TMD patients are difficult to manage and it is a therapeutic challenge due to multiple associated contributing factors ${ }^{5,6}$. It can be subdivided into two types: muscular and articular ${ }^{2,5}$. The difference between the two is crucial in the management. Intra-articular dysfunction of TMJ includes disc displacement with or without reduction which, if left untreated can progress to degenerative changes of the involved joint. Inflammation is one of the main cause among many underlying etiological factors that cause pain and dysfunction ${ }^{2,10}$. TMD patients have physical as well as psychological impact of the disease and their routine and quality of life is adversely affected ${ }^{6,11,12}$.

We found in our study that TMDs are more frequent in 30-40 years age group with mean age of 34 years. This finding is also reported in literature $7,11,13-15$. It was also observed in the study that majority of patients were females $(79 \%)$. This is evident in other studies as well7,9,14,15.

Limited mouth opening, pain on mouth opening and pain on chewing are the chief complaints of pati- ents suffering from TMDs. However, few patients also complain of clicking sounds 3,4 . Tozoglu et all4, observed otologic symptoms with TMJ pain in $29 \%$ of their patients, which may be due to close anatomical location of TMJ with ear and inflammation of TMJ or injury to retrodiscal tissue.

Conservative management is the first line of treatment which includes patient cognitive behavioural therapy, diet modification, jaw rest, exercises against res-

\begin{tabular}{|l|c|c|c}
\multirow{2}{*}{$\boldsymbol{p}$-value } & \multicolumn{3}{|c}{ Post-Operative } \\
\multirow{2}{*}{0.08} & Present & Absent & $\boldsymbol{p}$-value \\
\cline { 2 - 3 } & $6(29 \%)$ & $15(71 \%)$ & 0.01 \\
\hline
\end{tabular}

istence, analgesics, splint therapy, physiotherapy and transcutaneous electric nerve stimulation $3,4,6,7$. Conversely, refractory cases require surgical interventions from minimally invasive arthrocentesis, arthroscopy and intra-articular injections to aggressive surgeries like disc repair, discetomy or total joint replacement ${ }^{3,4}$. It is suggested in literature that $60-80 \%$ of patients benefit from conservative therapy ${ }^{2,5}$.

TMJ arthrocentesis is a minimally invasive treatment option between conservative management and invasive surgery. It is considered superior treatment option after the failure of conservative management of TMD patients5,10,13. It functions by flushing out inflammatory mediators, degraded proteins from superior joint space with sterile solution as they are the main cause of pain. It also disrupts immature adhesions which decrease negative pressure of the joint by reducing friction between intra-articular surfaces and increasing mandibular mobility 1,2,12. However its effect does not continue for long time as it does not rehabilitate the diseased structures of TMJ3. How much volume of solution is required to achieve the goal of arthrocentesis is still controversial. It is suggested in literature that 100 $\mathrm{ml}$ of fluid is ample for therapeutic lavage of TMJ. It is also proposed that ideal lavage volume for arthrocentesis should be $300-400 \mathrm{ml}^{1}$. However; Palma et al ${ }^{16}$, in his study conducted on cadaver hypothesized that 25 $\mathrm{ml}$ of lavage solution is sufficient enough for conventional arthrocentesis. Grossmann et al ${ }^{17}$ concluded that volume of arthrocentesis solution does not affect the outcome. As volume of solution for arthrocentesis is a controversial topic, type of solution to be used is not that debated. Ringers lactate or physiologic saline solutions are the two commonly used solutions for arthrocentesis. Soni ${ }^{1}$, proposed that articular disc tissues show 
better tolerance to Ringers lactate as it is close to human serum.

Intra-articular injection is among one of the therapy used in refractory cases of $\mathrm{TMD}^{5,11,18}$. PRP is a concentrate of growth factors which includes vascular endothelial growth factors, platelet derived growth factors and transforming growth factors B1, which are necessary for the cell production and extracellular matrix changes $3,4,9,19$. PRP improves joint function by restoring the productivity of synoviocytes for hyaluronic acid, stimulates production of cartilage by chondrocytes and improves angiogenesis and promotes stem cell migration ${ }^{3,18}$. The therapeutic content of PRP counts upto 1 million platelets per $1 \mathrm{ml}^{19}$.

Comparing the outcomes based on pain score of arthrocentesis alone and arthrocentesis with PRP showed that pre-operative pain score on VAS was $6.48 \pm$ 1.470 and $7.29 \pm 1.007$ which decreased to $1.81 \pm .602$ and $1.19 \pm 0.402$ respectively. Both groups showed significant gradual decrease in pain over time, this is in conformity by several authors in literature3,5,7,18. Intergroup comparison showed no statistically significant difference. It is evident in previous studies ${ }^{3,5}$.

Our study showed that in group I, mean MMO was $12.81 \pm 2.8$ which increased to $31.24 \pm 5.7$ and in group II, mean MMO was $12.71 \pm 3.0$ which increased to $39.43 \pm 3.2$. These values are similar to the statistics of study conducted by Rajputet $a l^{3}$. Mouth opening increased gradually in our both groups, this result is consistent with previous studies $5,7,18$. It was found in our study that PRP group showed better results, which is appreciated in other studies as well ${ }^{3,4}$. Khallaf ${ }^{13}$, in their study conducted on ${ }^{18}$, TMD patients performed arthrocentesis followed by two intra-articular PRP injections with the interval of three months and it was found that MMO increased by $8 \pm 1.5 \mathrm{~mm}$, which was highly significant. Increase in MMO could be due to growth factors which restore viscosity of synovial fluid and improves jaw movement ${ }^{7}$. Nabil et al ${ }^{5}$ in their comparative study conducted on 20 temporomandibular joints showed improvement in both groups after arthrocentesis and arthrocentesis with PRP but they didn't find statistically significant inter-group difference, which is in variance to our study results.

Clicking sounds were noticed in $69 \%$ of our patients and it reduced with treatment in both groups, which is supported by literature ${ }^{7,18}$. There was statistically significant difference between groups after the treatment $(p$-value $=0.01)$. It was found that PRP group showed more joint sound reduction than the compara- tive group, which corroborated our study3,4. Raed 11 , used PRP injections in the superior joint space of 34 patients with disc displacement without reduction and he noticed that joint clicking sound intensity decreased gradually over follow up. It is suggested that the optimal duration of PRP treatment is six months9,11.

Fernandez ${ }^{15}$, conducted a comparative study on TMDs patients with intra-articular PRP and Hyaluronate injection. It was found that PRP group showed better outcomes. Similarly, Sousa et al ${ }^{19}$, conducted study on eighty TMD patients with four different treatment modalities and it was concluded that patients who received PRP treatment benefitted the most of the four groups. Nitecka et $a^{20}$, used intra-muscular PRP in masseter muscle of myofascial pain patients and obtained satisfactory results. Similar further studies should be done to assess and evaluate the optimum frequency of PRP injections and whether PRP is dose dependant or not for the long term effects.

Ivask et al ${ }^{12}$ did a comparative study on botox with arthrocentesis and botox only to temporal and masseter muscle on 20 patients. Group with combination treatment showed more improvement in all variables. This study emphasized the significance of combination therapy in the management of refractory TMD. It also foregrounds the need of further studies on different combination therapies in the management of TMD patients.

\section{CONCLUSION}

Arthrocentesis with PRP is a simple and costeffective treatment method with promising results, which can be opted before more invasive surgical procedures. Gradual improvement was observed in both our groups but combination therapy group showed better results probably due to mechanical effects of arthrocentesis and regenerative properties of PRP.

\section{CONFLICT OF INTEREST}

This study has no conflict of interest to be declared by any author.

\section{REFERENCES}

1. Soni A. Arthrocentesis of temporomandibular joint-bridging the gap between non-surgical and surgical treatment. Ann Maxillofac Surg 2019; 9(1): 158-67.

2. Chandrashekhar VK, Kenchappa U, Chinnannavar SN, Singh S. Arthrocentesis a minimally invasive method for TMJ disc disorders-A prospective study. J Clin Diagn Res 2015; 9(10): ZC59-62.

3. Rajput A, Bansal V. A comparative analysis of intra-articular injection of platelet-rich plasma and arthrocentesis in temporomandibular joint disorders. J Maxillofac Oral Surg 2020; 21(2): 1-8.

4. Hanci M, Karamese M, Tosun Z, Murad TM, Duman S, Savaci N. Intra-articular platelet-rich plasma injection for the treatment of 
temporomandibular disorders and a comparison with arthrocentesis. J Cranio Maxillofac Surg 2015; 43(1): 162-66.

5. Nabil Y, Seleem H, Hassan N. Comparative study of the use of arthrocentesis and arthrocentesis in addition with platelet rich plasma in management of temporomandibular joint internal derangement. Egypt J Oral Maxillofac Surg 2019; 10(1): 9-19.

6. Pihut M, Szuta M, Ferendiuk E, Zenczak-Wieckiewicz D. Evaluation of pain regression in patients with temporomandibular dysfunction treated by intra-articular platelet rich plasma injections: a preliminary report. Biomed Res Int 2014; 132369(2014): 1-7.

7. Hassan EF, Ali TM, Abdulla N. The clinical efficiency of platelet rich plasma in the treatment of temporomandibular joint disorders. Alex Dent J 2016; 41(3): 226-31.

8. Nitzan DW, Dolwick MF, Martinez GA. Temporomandibular joint arthrocentesis: a simplified treatment for severe, limited mouth opening. J Oral Maxillofac Surg 1991; 49(11): 1163-70.

9. Hegab AF, Ali HE, Elmasry M, Khallaf MG. Platelet-rich plasma Injection as an effective treatment for temporomandibular joint osteoarthritis. J Oral Maxillofac Surg 2015; 73(9):1706-13.

10. Kütük N, Baş B, Kazan D, Yüceer E. Is repeated arthrocentesis beneficial in the treatment of temporomandibular disorders: A retrospective study. J Oral Maxillofac Surg 2019; 77(7): 1359-64.

11. Raed M, Alnuamy SH, Hamid FT, Azzamily TJ, Ismaeel SA, Sammir R, et al. The efficacy of platelets rich plasma injection in the superior joint space of the temporomandibular joint guided by ultra sound in patients with non-reducing disk displacement. J Maxillofac Oral Surg. 2017; 16(1): 43-47.

12. Ivask $O$, Leibur E, Akermann $S$, Tamme $T$, Voog-Oras Ü. Intramuscular botulinum toxin injection additional to arthrocentesis in the management of temporomandibular joint pain. Oral Surg Oral Med Oral Pathol Oral Radiol 2016; 122(4): e99-106.

13. Khallaf MG, Sidebottom AJ, Elrawy HF, Elmasry MS. Evaluation of platelet rich plasma effect on treatment of temporomandibular joint anterior disc displacement. Ameri J Oral Maxillofac Surg 2019; 6(1): 14-26.

14. Tozoglu S, Bayramoglu Z, Ozkan O. Outcome of otologic symptoms after temporomandibular joint arthrocentesis. J Craniofac Surg 2015; 26(4): e344-47.

15. Fernández-Ferro $M$, Fernández-Sanromán J, Blanco-Carrión A, Costas-López A, López-Betancourt A, Arenaz-Bua J, et al. Comparison of intra-articular injection of plasma rich in growth factors versus hyaluronic acid following arthroscopy in the treatment of temporomandibular dysfunction: A randomised prospective study. J Craniomaxillofac Surg 2017; 45(4): 449-54.

16. Palma LF, Rocha PR, Chaddad Neto FEA, Smith RL, de Moraes LOC. Irrigation fluid volume requirement for conventional arthrocentesis of the temporomandibular joint: a cadaver study. Int J Oral Maxillofac Surg 2020; 49(11): 1459-63.

17. Grossmann E, Poluha RL, Iwaki LCV, Iwaki Filho L. Arthrocentesis with different irrigation volumes in patients with disc displacement without reduction: One-year follow-up. Cranio 2020; 38(2): 122-27.

18. Zotti F, Albanese M, Rodella LF, Nocini PF. Platelet-rich plasma in treatment of temporomandibular joint dysfunctions: Narrative Review Int J Mol Sci 2019; 20(2): 277-32.

19. Sousa BMD, López-Valverde N, López-Valverde A, Caramelo F, Fraile JF, Payo JH, et al. Different treatments in patients with temporomandibular joint disorders: A comparative randomized study. Med 2020; 56(3): 113-18.

20. Nitecka-Buchta A,Walczynska-Dragon K, Kempa WM, Baron S. Platelet-rich plasma intramuscular injections - antinociceptive therapy in myofascial pain within masseter muscles in temporomandibular disorders patients: a pilot study front neurol. Front Neurol 2019; 19(10): 250. 